\title{
The influence of parental encouragement and caring about healthy eating on children's diet quality and body weights
}

\author{
Erin Faught, Kerry Vander Ploeg, Yen Li Chu, Kate Storey and Paul J Veugelers* \\ Population Health Intervention Research Unit, School of Public Health, University of Alberta, 3-50 University Terrace, \\ 8303-1 12 Street, Edmonton, Alberta, Canada, T6G 2T4
}

Submitted 22 December 2014: Final revision received 24 April 2015: Accepted 28 May 2015: First published online 23 June 2015

\begin{abstract}
Objective: In order to mitigate childhood obesity, evidence on what influences children's health behaviours is needed to inform new health promotion strategies. The present study investigated the association between parental practices and their child's diet and body weight status.

Design: Grade 5 students and their parents completed health surveys. Parents were asked how much they 'encourage their child to eat healthy foods' and how much they 'personally care about healthy eating'. Children's diet quality and vegetable and fruit intake were assessed using an FFQ. Children's heights and weights were measured to determine body weight status. Mixed-effects regression models were used to determine the influence of parental responses on the outcomes of interest.

Setting: Elementary schools across the province of Alberta, Canada.

Subjects: Grade 5 students (aged 10 and 11 years; $n$ 8388) and their parent(s).

Results: Most parents reported caring about healthy eating and encouraging their child to eat healthy foods at least quite a lot. Children whose parents who cared or encouraged 'very much' compared with 'quite a lot' were more likely have better diet quality and were less likely to be overweight. Children whose parents both cared and encouraged 'very much' compared with 'quite a lot' scored an average of $2 \cdot 06$ points higher on the diet quality index $(\beta=2 \cdot 06 ; 95 \%$ CI $1.45,2 \cdot 66)$.

Conclusions: Health promotion strategies that aim for a high level of parental interest and encouragement of their children to eat healthy foods may improve diet quality and prevent overweight among children.
\end{abstract}

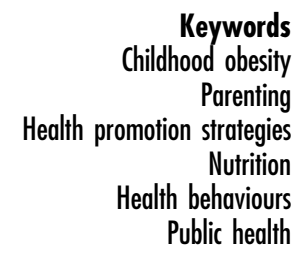

Childhood obesity is an important public health concern across the world, with rates increasing dramatically over recent decades in many developed countries ${ }^{(1-4)}$. A recent review has shown that in some European countries, these increasing trends have stabilized ${ }^{(1,3,4)}$. Childhood obesity can lead to insulin resistance and type 2 diabetes, hypertension and CVD later in life ${ }^{(5,6)}$.

Poor diet is widely acknowledged to be a key driver of childhood obesity. The most recent Canadian Community Health Survey reported that over $60 \%$ of children aged 9-13 were not consuming current Canadian recommendations for servings of vegetables and fruit ${ }^{(7)}$. Low daily consumption of vegetables and fruit is also common among children in other developed countries ${ }^{(8,9)}$. Higher consumption of nutritious, lower-energy foods, such as vegetables and fruit, is assumed to displace highly energydense, non-nutritious foods in children, assisting in weight management, although there is little empirical evidence to confirm this ${ }^{(10)}$. In adolescence, a period of rapid development, the consumption of vegetables and fruits is considered to be important ${ }^{(11)}$. Vegetable and fruit consumption in adulthood has been shown to be protective against CVD, cancer and stroke ${ }^{(2,11)}$. Because dietary habits learned in childhood and adolescence often persist into adulthood, healthy eating patterns established in childhood may lead to continued healthy behaviour in adulthood and prevention of lifetime disease $e^{(6,8,11)}$.

There is an extensive literature base providing strong evidence that parents play an important role in shaping their children's health behaviours and outcomes ${ }^{(4,12-16)}$. As such, parents are the focus of many public health strategies aiming to improve children's diet and reduce the prevalence of childhood obesity ${ }^{(2,17-20)}$. Recent systematic reviews have shown that parenting style, rules, home environment and parental role modelling are associated with their children's diet and body weight ${ }^{(4,12,15,16)}$. Similar findings have been found for other children's health behaviours such as television viewing and physical 
activity $^{(17,18,21)}$. The dietary literature about parental practices and children's outcomes predominantly focuses on specific dietary outcomes such as breakfast consumption, energy intake, soft drink consumption, and vegetable and fruit consumption ${ }^{(11-14)}$. Although these specific dietary behaviours can act as a proxy for overall adequacy of the diet, studies linking parental practices to complete dietary patterns are limited. The use of a diet quality index allows an assessment of the whole diet pattern rather than specific foods or nutrients, allowing the evaluation of how overall diet patterns align with recommendations ${ }^{(22,23)}$. In children, higher scores on diet quality indices have been shown to be associated with better lean mass, improved school performance and reduced feelings of sadness and worry, although studies using diet quality indices in children are $\mathrm{few}^{(23-25)}$. In adults, diet quality indices have been used to determine chronic disease risk and risk of mortality from chronic disease ${ }^{(22)}$. Although there is much literature to demonstrate that parents exert a key influence on children's specific nutrition behaviours, studies linking parental encouragement and caring about healthy eating and its influence on children's diet quality are limited. As diet quality indices provide a more comprehensive overview of dietary patterns that better predict disease risk than individual nutrients, foods or habits, and studies using diet quality indices in children are limited, investigating determinants of overall diet quality for children can make an important contribution to the children's health literature ${ }^{(22,23,26)}$.

To inform and improve the effectiveness of policies and programmes for the improvement of children's diets and prevention of childhood obesity and chronic disease, we investigated the association between parental encouragement and caring about healthy eating and their child's diet quality and body weight status using a large, populationbased sample of parents and children in Alberta, Canada.

\section{Methods}

\section{Study design}

The present research is an analysis of secondary data acquired from the Raising healthy Eating and Active Living (REAL) Kids Alberta Evaluation, a population-based survey of grade 5 children (aged 10-11 years) and their parents or guardians throughout the province of Alberta, Canada. The evaluation serves to assess the impact of programmes and policies for the prevention of childhood obesity administered by Alberta Health. The survey occurred in three waves in 2008, 2010 and 2012 using a one-stage stratified random sampling design ${ }^{(21)}$. All Albertan schools were included in the sampling frame with the exception of private, francophone, on-reserve federal, charter and colony schools ${ }^{(21)}$. This accounted for $90.2 \%$ of eligible students in the province. Schools were stratified into three geographical areas - metropolitan, urban and rural - and schools were randomly selected within these strata to achieve a balance of students from each group ${ }^{(21)}$. Upon selection, the school principal was given the opportunity to agree or decline to his/her school's participation. If the principal declined, another school within the stratum was randomly selected as a replacement until the target of approximately 150 schools in each year was reached. All three waves had the same protocol and reached a new group of parents and their grade 5 children. The evaluation consisted of three questionnaires: one directed at parents or guardians (home survey) and two directed at children (student survey and FFQ). Informed consent was provided by parents at the time of the home survey. Students provided assent before the completion of their surveys. Trained evaluation assistants administered the student survey and FFQ, as well as collected height and weight measurements, during classroom time at the participating schools. Additional information about the REAL Kids Alberta Evaluation can be accessed at the website (http://www.realkidsalberta.ca). All procedures involving human subjects were approved by the University of Alberta's Health Research Ethics Board. Ethical approval was also obtained for the current secondary data analysis from the University of Alberta's Health Research Ethics Board.

\section{Assessment of exposure}

The home survey directed at parents or guardians of students included two questions related to beliefs and support for healthy eating: (i) 'How much do you personally care about eating healthy foods?' and (ii) 'To what extent do you encourage your child to eat healthy foods?' Responses for each question corresponded to an ordinal scale from 1 to 4 , where a response of $1=$ 'not at all', $2=$ 'a little bit', $3=$ 'quite a lot' and $4=$ 'very much'. Few parents responded 'not at all' or 'a little bit' to either question (Table 1) and therefore these responses were combined into one category for the purposes of analysis. Surveys are available online (http://www.realkidsalberta.ca).

\section{Assessment of outcome measures}

\section{Diet quality}

Diet quality was assessed using the Diet Quality IndexInternational (DQI) score calculated based on student responses to the Harvard Youth/Adolescent FFQ. The DQI is a composite measure of diet that ranges from 0 to 100 , with higher scores indicating better diet quality. It measures diet quality over four categories: adequacy, variety, moderation and overall balance ${ }^{(26)}$. A DQI score is based on a combination of consumption of whole foods and nutrients that can be derived from responses to the FFQ.

\section{Meeting recommendations for vegetables and fruit}

Each participant's vegetable and fruit intake was calculated as number of servings from the 'Vegetables and Fruit' 
category outlined by Eating Well with Canada's Food Guide $^{(27)}$ as also measured using the FFQ. A binary variable was generated for each participant to indicate if he/she met or did not meet the recommendation of six servings of vegetables and fruit daily ${ }^{(27)}$.

\section{Weight status}

Height was measured to the nearest $0 \cdot 1 \mathrm{~cm}$ using a stadiometer (Seca-Stadiometers, Germany) and body weight to the nearest $0 \cdot 1 \mathrm{~kg}$ using calibrated digital scales (Health-o-meter, USA). Children were asked to remove their shoes for both measurements. BMI was calculated as $\mathrm{kg} / \mathrm{m}^{2}$ and overweight was defined using the BMI cut-off point for children and youth established by the International Obesity Task Force ${ }^{(28)}$. This cut-off point is based on the adult health-related definition of overweight $\left(B M I \geq 25 \mathrm{~kg} / \mathrm{m}^{2}\right.$ ), but is adjusted for specific age and sex categories for children.

\section{Confounding variables}

Analyses were adjusted for child's gender, school region, year of evaluation, parental education attained and household income. Tables show results for unadjusted, partially adjusted and fully adjusted models. Analyses were also adjusted for energy intake for improved validity as per recommendations for the analyses of FFQ data ${ }^{(29)}$. Energy intake was also derived from the FFQ.

\section{Analysis}

\section{Sample characteristics}

A total of 15875 parents and students were invited to participate; 9784 parents completed and returned their home survey as well as provided consent for their child to participate, resulting in a $62 \%$ consent rate. Following receipt of their parents' consent, 9210 children participated in the evaluation resulting in a $58 \%$ completion rate. Students or parents who had incomplete or missing responses for any of the variables of interest were excluded from the analysis, as were students reporting energy intakes lower than $2092 \mathrm{~kJ}$ ( $500 \mathrm{kcal}$ ) or higher than $20920 \mathrm{~kJ}$ (5000 kcal), leaving 8388 parents and students to be included in the current analysis.

\section{Statistical analysis}

Random-effects models were applied due to the clustering of students within schools. Univariable linear and logistic regression models were first used to quantify associations between parental encouragement and caring variables and the outcomes of diet quality, meeting vegetable and fruit requirements and overweight. The response of 'quite a lot' was used as a reference category. Next, we applied multivariable linear and logistic regression models to adjust for potential confounders including child gender, energy intake, school region, year of evaluation, parental education and household income (model 1). Finally, we considered all exposures simultaneously to determine their independent associations with the outcomes of interest (model 2).

To determine if parental encouragement and personal caring about healthy eating had a synergistic effect on the outcomes of interest, an interaction model was built. Parents who responded 'very much' to both questions or 'not at all/a little bit' to both questions were compared with parents who responded 'quite a lot' to both questions.

All analyses were completed using the statistical software package Stata SE version 13.

\section{Results}

Descriptive statistics for children and parents are presented in Table 1. Because of infrequency of the response of 'not at all', these responses were combined with response of 'a little bit' for the purposes of subsequent analysis.

Parental encouragement and personal caring about healthy eating were both associated with diet quality. Children whose parents encouraged them 'very much' to eat healthy foods compared with those whose parents

Table 1 Characteristics of grade 5 students and their parent(s) or guardian(s) participating in the REAL Kids Alberta Evaluation in Alberta, Canada in 2008, 2010 and 2012 ( $n$ 8388)

\begin{tabular}{|c|c|}
\hline Characteristic & Value \\
\hline \multicolumn{2}{|l|}{ Characteristics of children } \\
\hline $\begin{array}{l}\text { Meets the daily recommended servings of } \\
\text { vegetables and fruit (\%) }\end{array}$ & $33 \cdot 3$ \\
\hline $\begin{array}{l}\text { Median no. of servings of vegetables and } \\
\text { fruit daily }\end{array}$ & $4 \cdot 15$ \\
\hline \multicolumn{2}{|l|}{ DQI score } \\
\hline Mean & $62 \cdot 5$ \\
\hline $95 \% \mathrm{Cl}$ & $62 \cdot 3,62 \cdot 8$ \\
\hline Mean age (years) & $10 \cdot 91$ \\
\hline Proportion male (\%) & 48.4 \\
\hline Overweight (including obese; \%) & $27 \cdot 8$ \\
\hline Obese (\%) & 7.6 \\
\hline \multicolumn{2}{|l|}{ Characteristics of parents or guardians } \\
\hline \multicolumn{2}{|l|}{ Encourage their child to eat healthy (\%) } \\
\hline Not at all & 0.2 \\
\hline A little bit & $6 \cdot 7$ \\
\hline Quite a lot & $47 \cdot 6$ \\
\hline Very much & 45.5 \\
\hline \multicolumn{2}{|l|}{ Personally care about eating healthy foods (\%) } \\
\hline Not at all & 0.4 \\
\hline A little bit & $10 \cdot 2$ \\
\hline Quite a lot & $51 \cdot 3$ \\
\hline Very much & 38.1 \\
\hline \multicolumn{2}{|l|}{ Educational attainment (\%) } \\
\hline Secondary or less & $25 \cdot 9$ \\
\hline College diploma & 41.1 \\
\hline University or graduate degree & 33.0 \\
\hline \multicolumn{2}{|l|}{ Household income (\$CAN; \%) } \\
\hline$\leq 50000$ & $24 \cdot 4$ \\
\hline $50001-75000$ & 17.5 \\
\hline $75001-100000$ & $20 \cdot 6$ \\
\hline$\geq 100001$ & 37.5 \\
\hline
\end{tabular}

DQI, Diet Quality Index-International. 
Table 2 Association of parental encouragement and personal caring about healthy eating with their grade- 5 child's diet quality and likelihood of meeting vegetable and fruit recommendations; REAL Kids Alberta Evaluation in Alberta, Canada in 2008, 2010 and 2012 ( $n$ 8388)

\begin{tabular}{|c|c|c|c|c|c|c|}
\hline & & & \multicolumn{4}{|c|}{ Multivariable } \\
\hline & \multicolumn{2}{|c|}{ Univariable } & \multicolumn{2}{|c|}{ Model 1† } & \multicolumn{2}{|r|}{ Model $2 \ddagger$} \\
\hline & $\beta / \mathrm{OR}$ & $95 \% \mathrm{Cl}$ & $\beta / \mathrm{OR}$ & $95 \% \mathrm{Cl}$ & $\beta / \mathrm{OR}$ & $95 \% \mathrm{Cl}$ \\
\hline \multicolumn{7}{|l|}{ DQI } \\
\hline \multicolumn{7}{|c|}{ Encourage their child to eat healthy foods } \\
\hline Very much & $1.55^{\star \star}$ & $1 \cdot 27,2 \cdot 19$ & $1.44^{\star \star}$ & $0.96,1.91$ & $0.79^{*}$ & $0.27,1.30$ \\
\hline Quite a lot & \multicolumn{2}{|c|}{ Reference } & \multicolumn{2}{|c|}{ Reference } & \multicolumn{2}{|r|}{ Reference } \\
\hline Not at all/A little bit & $-1 \cdot 73^{\star \star}$ & $-2 \cdot 61,-0.84$ & $-1.52^{\star \star}$ & $-2 \cdot 37,-0.67$ & $-1.01^{*}$ & $-1.96,-0.62$ \\
\hline \multicolumn{7}{|c|}{ Personally care about eating healthy foods } \\
\hline Very much & $1.73^{* *}$ & $1 \cdot 28,2 \cdot 19$ & $1 \cdot 66^{\star *}$ & $1 \cdot 09,2 \cdot 22$ & $1 \cdot 24^{\star *}$ & $0.64,1.84$ \\
\hline Quite a lot & \multicolumn{2}{|c|}{ Reference } & \multicolumn{2}{|c|}{ Reference } & \multicolumn{2}{|r|}{ Reference } \\
\hline Not at all/A little bit & $-1 \cdot 55^{\star \star}$ & $-2.27,-0.82$ & $-1 \cdot 44^{*}$ & $-2 \cdot 26,-0.63$ & $-1.06^{\star}$ & $-1.97,-0.16$ \\
\hline \multicolumn{7}{|c|}{ Likelihood of meeting vegetable and fruit recommendations } \\
\hline Very much & $1.40^{* *}$ & $1.28,1.54$ & $1.42^{\star \star}$ & $1.27,1.60$ & $1 \cdot 24^{\star *}$ & $1.12,1.39$ \\
\hline Quite a lot & \multicolumn{2}{|c|}{ Reference } & \multicolumn{2}{|c|}{ Reference } & \multicolumn{2}{|r|}{ Reference } \\
\hline Not at all/A little bit & $0.74^{*}$ & $0.60,0.91$ & 0.79 & $0.60,1.03$ & 0.86 & $0.65,1 \cdot 15$ \\
\hline \multicolumn{7}{|c|}{ Personally care about eating healthy foods } \\
\hline Very much & $1.42^{* \star}$ & $1.29,1.57$ & $1.44^{\star \star}$ & $1 \cdot 28,1.65$ & $1 \cdot 31^{\star *}$ & $1.15,1.49$ \\
\hline Quite a lot & \multicolumn{2}{|c|}{ Reference } & \multicolumn{2}{|c|}{ Reference } & \multicolumn{2}{|r|}{ Reference } \\
\hline Not at all/A little bit & $0.81^{*}$ & $0.68,0.95$ & $0.78^{*}$ & $0.63,0.95$ & 0.84 & $0.68,1.03$ \\
\hline
\end{tabular}

DQI, Diet Quality Index-International.

${ }^{\star} P<0.05,{ }^{* \star} P \leq 0.01$.

†Model 1 adjusted for child gender, energy intake, parental education, household income, school region and year of participation.

¥Model 2 adjusted for child gender, energy intake, parental education, household income, school region, year of participation, parental encouragement of healthy eating and parent personally caring about healthy eating.

encouraged them 'quite a lot' scored 1.44 (95\% CI 0.96 , 1.91) DQI units higher, adjusting for relevant confounders (model 1, Table 2). This association remained statistically significant when parental caring about healthy foods was included in the model (model 2, Table 2). Children whose parents encouraged their child 'not at all' or 'a little bit' to eat healthy foods scored 1.52 (95\% CI -2.37, -0.67) DQI units lower compared with those whose parents encouraged them 'quite a lot' (model 1, Table 2). These associations were very similar for responses about parental caring about healthy eating (Table 2). Children whose parents personally cared 'very much' about healthy eating scored 1.66 (95\% CI 1.09, 2.22) DQI units higher than those whose parents cared 'quite a lot', while those who cared 'not at all' or 'a little bit' scored 1.44 (95\% CI $-2 \cdot 26,-0 \cdot 63$ ) DQI units less (Table 2). These associations remained significant when including parental encouragement in the model.

Increased parental encouragement and caring about healthy eating were associated with increased likelihood of their child meeting recommendations for vegetables and fruit (Table 2). Children whose parents encouraged them 'very much' to eat healthy foods were 1.42 (95\% CI $1.27,1.60)$ times more likely to meet vegetable and fruit recommendations than those whose parents encouraged them 'quite a lot', when considering relevant confounders (Table 2). Children whose parents cared about healthy eating 'very much' compared with those who cared 'quite a lot' were 1.44 (95\% CI $1.28,1.65)$ times more likely to meet vegetable and fruit recommendations than those who cared 'quite a lot'. The association between parents who encouraged and cared about healthy eating 'not at all' or 'a little bit' and likelihood of meeting vegetable and fruit recommendations was weak, but the trend did show a gradient that less encouragement and caring led to lower likelihood of meeting recommendations (Table 2).

Children whose parents encouraged them 'very much' to eat healthy foods were 0.83 (95\% CI $0.71,0.96)$ times more likely to be overweight (model 1, Table 3). When parental caring about healthy eating was included in the model, this association remained significant (model 2, Table 3). No statistically significant association was observed between parental caring about healthy eating and child likelihood of overweight when relevant confounders were considered.

Further analysis revealed a clear synergistic and statistically significant interaction between parental encouragement for healthy eating and parental caring about healthy eating for children's dietary outcomes. Compared with children whose parents responded 'quite a lot' for both questions, children whose parents who responded 'very much' to both questions scored an average of 2.06 (95\% CI 1.45, 2.66) DQI units higher. Comparatively, those whose parents responded 'not at all' or 'a little bit' to both questions scored an average of 1.77 (95\% CI $-2.93,-0.61)$ fewer DQI units. Children whose parents responded 'very much' to both questions compared with the reference of 'quite a lot' for both had 1.65 (95\% CI 1.41, 1.93) times the odds of meeting vegetable and fruit requirements. There was a decreased, although not statistically significant, likelihood of child 
Table 3 Association of parental encouragement and personal caring about healthy eating with their grade- 5 child's likelihood of overweight (including obese); REAL Kids Alberta Evaluation in Alberta, Canada in 2008, 2010 and 2012 ( $n$ 8388)

\begin{tabular}{|c|c|c|c|c|c|c|}
\hline & & & \multicolumn{4}{|c|}{ Multivariable } \\
\hline & \multicolumn{2}{|c|}{ Univariable } & \multicolumn{2}{|c|}{ Model $1 \dagger$} & \multicolumn{2}{|c|}{ Model 2‡ } \\
\hline & OR & $95 \% \mathrm{Cl}$ & OR & $95 \% \mathrm{Cl}$ & OR & $95 \% \mathrm{Cl}$ \\
\hline \multicolumn{7}{|c|}{ Encourage their child to eat healthy foods } \\
\hline Very much & $0.74^{\star *}$ & $0.68,0.82$ & $0.83^{*}$ & $0.71,0.96$ & $0.82^{*}$ & $0.69,0.98$ \\
\hline Quite a lot & \multicolumn{2}{|c|}{ Reference } & \multicolumn{2}{|c|}{ Reference } & \multicolumn{2}{|c|}{ Reference } \\
\hline Not at all/A little bit & 0.99 & $0.82,1.19$ & 0.94 & $0.72,1.23$ & 0.94 & $0.71,1 \cdot 23$ \\
\hline \multicolumn{7}{|c|}{ Personally care about eating healthy foods } \\
\hline Very much & $0.81^{* *}$ & $0.73,0.90$ & 0.94 & $0.81,1.08$ & 1.02 & $0.87,1 \cdot 20$ \\
\hline Quite a lot & \multicolumn{2}{|c|}{ Reference } & \multicolumn{2}{|c|}{ Reference } & \multicolumn{2}{|c|}{ Reference } \\
\hline Not at all/A little bit & 1.08 & $0.92,1.26$ & 1.06 & $0.87,1.30$ & 1.05 & $0.85,1.30$ \\
\hline
\end{tabular}

${ }^{\star} P<0.05,{ }^{\star \star} P \leq 0.01$

†Model 1 adjusted for child gender, parental education, household income, school region and year of participation.

¥Model 2 adjusted for child gender, parental education, household income, school region, year of participation, parental encouragement of healthy eating and parent personally caring about healthy eating.

overweight for responses of 'very much' for both questions $(\mathrm{OR}=0.85 ; 95 \%$ CI $0.72,1 \cdot 01)$.

\section{Discussion}

In the present study we assessed the association of parental encouragement and caring about healthy eating with children's diet and body weight status. Using a large, population-based sample of Canadian children, we showed that increased parental encouragement and caring were both independently associated with increases in diet quality and likelihood of meeting recommendations for vegetable and fruit consumption. The existing literature has revealed positive impacts of parental involvement on children's consumption of vegetables and fruit ${ }^{(8)}$. However, to our knowledge, the present study is the first to reveal a correlation of parent caring and encouragement with healthy eating and diet quality of children. We also found that increased parental encouragement for their child to eat healthy foods was associated with a decreased likelihood of child overweight. Moreover, we showed that parental encouragement and caring about healthy eating work synergistically on the diet quality of children.

The majority of parents in this Canadian sample reported encouraging and personally caring about healthy eating at least 'quite a lot'. This is similar to a Dutch study in which parents completed eight questions related to encouragement of their child's eating habits (e.g. 'I encourage my child to enjoy his/her food') ${ }^{(3,30)}$. On a 5-point Likert scale, the mean score for encouragement of positive eating behaviours was $3 \cdot 88$, indicating that the parents in this sample encouraged their children to exhibit positive eating habits often ${ }^{(3,31-33)}$. Ohly et al. measured parental caring about food and eating in British parents using a Food Involvement Score and found a mean score of 47 out of a possible 60 in their sample, again similar to the high level of caring observed in our sample ${ }^{(8,11)}$. Parents in the 2008 and 2010 REAL Kids Alberta Evaluation sample reported higher encouragement and personal caring about healthy eating than about physical activity, with $23 \cdot 1 \%$ of parents reporting that they personally cared about physical activity 'not at all' or 'a little bit' ${ }^{,(3,44)}$. This may indicate that parents may be more open to health promotion programmes that target improvement in eating behaviours compared with programmes that target other health behaviours. Alternatively, parents may be more aware of the relationship between diet and health than they are about physical activity.

The present study's findings are consistent with existing research indicating that parental encouragement and caring about healthy eating are associated with improved dietary outcomes for children ${ }^{(8,11,13,14,35)}$. In a longitudinal study following Dutch parents and their children from age 6 to 8 years, parental encouragement about eating was found to be positively related to fruit intake ${ }^{(2)}$. Data collected from parents and children ranging from 18 months to 5 years of age in the UK also demonstrated a link between parental food involvement and vegetable and fruit intake in their children ${ }^{(8)}$. The same study demonstrated that increased parental involvement, or personal caring, about food improved their own diet ${ }^{(8,19)}$. Improvements in parental diet resulted in improved parental behaviour modelling, which reviews have found to be an important predictor of vegetable and fruit intake in children and adolescents ${ }^{(15,19,30)}$. Additionally, parents have frequently cited in qualitative studies that a healthy child is the primary motivator for household food practices $^{(17,31-33)}$. Findings from Project EAT (Eating Among Teens), a cross-sectional study using a large sample of American adolescents, showed that availability of foods in the home is among the most important determinants of children's diets ${ }^{(4,11,36)}$. The quantitative findings from the present study complement the above findings and add an association with overall diet quality beyond single nutrients, foods or behavioural indices. 
It is important to note that we observed significant improvements in all observed health outcomes with an incremental change in response from 'quite a lot' to 'very much'. This may indicate that consistent caring and encouragement by parents has a pronounced benefit to children's health over moderate expression of these behaviours. We also saw that the incremental changes worked synergistically for encouragement and parental caring: when combined, the positive impact on children's health outcomes was larger. These findings seem consistent with the existing literature about parenting style. Reviews about the association between parenting style and child overweight indicate that permissive parenting styles (i.e. parents who are not demanding of their child but highly responsive to their needs) and authoritarian parenting styles (i.e. parents who are highly demanding and less responsive to their children) are both associated with childhood overweight ${ }^{(3,4,13,14)}$. Meanwhile, authoritative parents who are both moderately responsive and demanding have children with the least likelihood of overweight ${ }^{(3,4)}$. This suggests that parents' personal beliefs about food and the action of those beliefs through encouragement, rather than dictation, for their children work together in influencing children's eating practices.

The present study has implications for health promotion strategies and interventions. The study reinforces previous evidence-based recommendations that parents are important health promotion targets for the prevention of childhood obesity and improvement of children's diets. Findings from Project EAT lead to the recommendation that parents both model and encourage healthful eating behaviours without focusing on weight to obtain a healthy weight as well as a positive body image ${ }^{(11,13,20,37)}$. When reviewing the literature about parenting and childhood obesity, Lindsay et al. found that parents play a key role in the prevention of childhood obesity not only in terms of their influence on dietary habits but also in their influence on physical activity and sedentary activities ${ }^{(19)}$. The authors further stated that existing data on interventions that include parents show promising results in the prevention of childhood obesity, although further research in this area is recommended ${ }^{(19)}$. Our work answers this call for research. Our findings inform interventions to prioritize healthy eating and prevent childhood obesity. We showed that parental caring about healthy eating plays a role in children's dietary habits and body weight, which may be reflected through improved home availability of healthy foods. Parental encouragement of healthy eating may add to this improvement in health behaviours and outcomes by influencing what children choose. Although we recommend parents as important targets for health promotion strategies, we acknowledge that the improvement of children's diets and preventing childhood obesity require an integrated, broad approach.

The REAL Kids Alberta Evaluation provides a large, representative sample of children, generalizability of results, directly measured heights and weights and a relatively high response rate, which should be considered as strengths. The usage of an FFQ allowed us to calculate a diet quality index which is reflective of overall disease risk due to diet. The findings from the present study are also underpinned by a robust, widely used theory regarding decision making and behaviours. The Theory of Planned Behavior $^{(36)}$ states that beliefs and actions founded on those beliefs predict behavioural intention, the most important predictor of a behaviour ${ }^{(17)}$. In the present study, we can deduce that positive parental support towards healthy eating is predictive of parents' behaviour; that is, provision of healthy food opportunities for children. The cross-sectional design of the research is a limitation to interpretations of causality. Additionally, self-reported survey data are subject to error and the health behaviours in question are prone to social desirability bias. The high prevalence of parental encouragement and caring about healthy eating may be indicative of volunteer bias and not be representative of subgroups that may not be able to prioritize or access healthy eating opportunities such as socio-economically disadvantaged populations. It has also been documented that parenting practices and styles are defined inconsistently in the literature and measured using a wide variety of tools ${ }^{(4,37)}$. As such, comparisons of our findings to existing parenting literature may be challenging. Future study on this topic should include home availability of vegetables and fruit, which may be an intermediate variable in the relationship between parental encouragement and caring with their child's diet and body weight ${ }^{(11,13,38)}$. Future studies should also include more components of parental practices in regard to eating. The present study was limited to the two components included.

\section{Conclusions}

The present research showed that parental encouragement and caring about healthy eating have important implications for body weight status and children's diet, most notably an improvement in overall diet quality. Further, the present research demonstrates that even when parents report a high prevalence of moderate to high caring and encouragement, the highest reported practices are associated with the most benefit to children. Health promotion initiatives that engage parents to consistently and effectively encourage and care about healthy eating may result in improvement of dietary outcomes of children and reduction of the prevalence of childhood obesity.

\section{Acknowledgements}

Acknowledgements: The authors thank all students, parents and schools for participating in the study. They also thank 
all evaluation assistants and health promotion coordinators for collecting and processing data; and Connie Lu, Pamela Bailey, Megan Purcell, Shelby Corley and Wendy Davis for leading the data collection, management and validation. Financial support: The REAL Kids Alberta Evaluation was funded through a contract with Alberta Health. The contract with Alberta Health supported data collection but had no role in the design, analysis or writing of this article. The present research was further funded through a Canada Research Chair in Population Health, an Alberta Research Chair in Nutrition and Disease Prevention, and an Alberta Innovates Health Solutions Scholarship to P.J.V. These sources of funding did not have a role in the design, analysis or writing of this article. All interpretations and opinions in the present study are those of the authors. Conflict of interest: None. Authorship: E.F., lead author of the paper, conducted all analyses and led all literature search, writing and interpretations. K.V.P. provided key support in conducting analyses and structuring the writing of the paper. Y.L.C. provided key support in conducting analyses, assisting in interpretation and provided extensive feedback for writing. K.S. assisted in the development of the research question, design of the study, in interpretation and providing feedback; and serves on the supervisory committee for E.F.'s studentship. P.J.V., Principal Investigator for the REAL Kids Alberta Evaluation which provided the data for this secondary analysis, played a key role in the development of the research question, design of the study, interpretation of results, structuring and writing of the manuscript; and serves as supervisor for E.F.'s studentship. Etbics of buman subject participation: All procedures involving human subjects were approved by the University of Alberta's Health Research Ethics Board. Informed, written consent for both parental and child participants was obtained from participating parents, written assent was obtained from all participating children.

\section{References}

1. Lien N, Henriksen HB, Nymoen LL et al. (2010) Availability of data assessing the prevalence and trends of overweight and obesity among European adolescents. Public Health Nutr 13, 1680-1687.

2. Wright K, Norris K, Newman Giger J et al. (2012) Improving healthy dietary behaviors, nutrition knowledge, and selfefficacy among underserved school children with parent and community involvement. Child Obes 8, 347-356.

3. Sleddens EFC, Kremers SPJ, Stafleu A et al. (2014) Food parenting practices and child dietary behavior. Prospective relations and the moderating role of general parenting. Appetite 79, 42-50.

4. Ventura AK \& Birch LL (2008) Does parenting affect children's eating and weight status? Int J Behav Nutr Phys Act 5, 15.

5. Singh AS, Mulder C, Twisk JWR et al. (2008) Tracking of childhood overweight into adulthood: a systematic review of the literature. Obes Rev 9, 474-488.

6. Roberts K, Shields M, de Groh M et al. (2012) Overweight and obesity in children and adolescents: results from the
2009 to 2011 Canadian Health Measures Survey, http://www. statcan.gc.ca/pub/82-003-x/2012003/article/11706-eng.htm (accessed February 2014).

7. Shields M \& Rozin P (2006) Overweight and obesity among children and youth. http://www.geog.ubc.ca/courses/geob370/ students/class10/ddchan/www/images/docs/Shields.pdf (accessed February 2014).

8. Ohly H, Pealing J, Hayter AKM et al. (2013) Parental food involvement predicts parent and child intakes of fruits and vegetables. Appetite 69, 8-14.

9. Berge JM, Wall M, Larson $\mathrm{N}$ et al. (2014) Youth dietary intake and weight status: healthful neighborhood food environments enhance the protective role of supportive family home environments. Health Place 26, 69-77.

10. Bayer O, Nehring I, Bolte G et al. (2014) Fruit and vegetable consumption and BMI change in primary school-age children: a cohort study. Eur J Clin Nutr 68, 265-270.

11. Neumark-Sztainer D, Wall M, Perry C et al. (2003) Correlates of fruit and vegetable intake among adolescents. Findings from Project EAT. Prev Med 37, 198-208.

12. Sleddens EFC, SMPL Gerards, Thijs C et al. (2011) General parenting, childhood overweight and obesity-inducing behaviors: a review. Int J Pediatr Obes 6, e12-e27.

13. Verloigne M, Van Lippevelde W, Maes L et al. (2012) Family- and school-based correlates of energy balancerelated behaviours in 10-12-year-old children: a systematic review within the ENERGY (EuropeaN Energy balance Research to prevent excessive weight Gain among Youth) project. Public Health Nutr 15, 1380-1395.

14. Van Lippevelde W, Velde Te SJ, Verloigne $M$ et al. (2013) Associations between family-related factors, breakfast consumption and BMI among 10- to 12-year-old European children: the cross-sectional ENERGY-study. PLOS ONE $\mathbf{8}$, e79550.

15. Webber KJ \& Loescher LJ (2013) A systematic review of parent role modeling of healthy eating and physical activity for their young African American children. J Spec Pediatr Nurs 18, 173-188.

16. McClain AD, Chappuis C, Nguyen-Rodriguez ST et al. (2009) Psychosocial correlates of eating behavior in children and adolescents: a review. Int J Behav Nutr Phys Act 6, 54.

17. Andrews KR, Silk KS \& Eneli IU (2010) Parents as health promoters: a theory of planned behavior perspective on the prevention of childhood obesity. J Health Commun 15, 95-107.

18. Vander Ploeg KA, Wu B, McGavock J et al. (2012) Physical activity among Canadian children on school days and nonschool days. J Phys Act Health 9, 1138-1145.

19. Lindsay AC, Sussner KM, Kim J et al. (2006) The role of parents in preventing childhood obesity. Future Child 16, 169-186.

20. Neumark-Sztainer D (2005) Preventing the broad spectrum of weight-related problems: working with parents to help teens achieve a healthy weight and a positive body image. J Nutr Educ Behav 37, Suppl. 2, S133-S140.

21. Fung C, Kuhle S, Lu C et al. (2012) From 'best practice' to 'next practice': the effectiveness of school-based health promotion in improving healthy eating and physical activity and preventing childhood obesity. Int J Behav Nutr Phys Act 9, 27 .

22. Marshall S, Burrows T \& Collins CE (2014) Systematic review of diet quality indices and their associations with health-related outcomes in children and adolescents. J Hum Nutr Diet 27, 577-598.

23. Smithers LG, Golley RK, Brazionis L et al. (2011) Characterizing whole diets of young children from developed countries and the association between diet and health: a systematic review. Nutr Rev 69, 449-467.

24. Florence MD, Asbridge M \& Veugelers PJ (2008) Diet quality and academic performance. $J$ Sch Health 78, 209-215. 
25. McMartin SE, Willows ND, Colman I et al. (2013) Diet quality and feelings of worry, sadness or unhappiness in Canadian children. Can I Public Health 104, e322-e326.

26. Kim S, Haines PS, Siega-Riz AM et al. (2003) The Diet Quality Index-International (DQI-I) provides an effective tool for cross-national comparison of diet quality as illustrated by China and the United States. J Nutr 133, 3476-3484.

27. Health Canada (2011) Eating Well with Canada's Food Guide. http://www.hc-sc.gc.ca/fn-an/food-guide-aliment/ index-eng.php (accessed April 2014).

28. Cole TJ, Bellizzi MC, Flegal KM et al. (2000) Establishing a standard definition for child overweight and obesity worldwide: international survey. BMJ 320, 1240-1243.

29. Willett W (2013) Nutritional Epidemiology, 3rd ed. New York: Oxford University Press.

30. McClain AD, Pentz MA, Nguyen-Rodriguez ST et al. (2011) Measuring the meanings of eating in minority youth. Eat Behav 12, 277-283

31. Devine CM, Jastran M, Jabs J et al. (2006) 'A lot of sacrifices': work-family spillover and the food choice coping strategies of low-wage employed parents. Soc Sci Med 63, 2591-2603.

32. Johnson CM, Sharkey JR \& Dean WR (2011) It's all about the children: a participant-driven photo-elicitation study of
Mexican-origin mothers' food choices. BMC Womens Health 11, 41.

33. Daniels SR, Jacobson MS, McCrindle BW et al. (2009) American Heart Association Childhood Obesity Research Summit: executive summary. Circulation 119, 2114-2123.

34. Vander Ploeg KA, Maximova K, Kuhle S et al. (2012) The importance of parental beliefs and support for physical activity and body weights of children: a population-based analysis. Can J Public Health 103, e277-e281.

35. Wrotniak BH, Epstein LH, Paluch RA et al. (2005) The relationship between parent and child self-reported adherence and weight loss. Obes Res 13, 1089-1096.

36. Azjen I (1985) From intentions to actions: a theory of planned behavior. In Action-Control: From Cognition to Behavior, pp. 11-39 [J Kuhl and J Beckman, editors]. Heidelberg: Springer.

37. Baranowski T, O'Connor T, Hughes S et al. (2013) Houston... We have a problem! Measurement of parenting. Child Obes 9. Suppl, S1-S4.

38. Rosenkranz RR \& Dzewaltowski DA (2008) Model of the home food environment pertaining to childhood obesity. Nutr Rev 66, 123-140. 